\title{
COVID-19 Pneumonia with Delayed Viral Clearance in a Patient with Active Drug-resistant Pulmonary Tuberculosis
}

\author{
Utpal Sarma ${ }^{1}$, Vipul Mishra ${ }^{2}$, Jyoti Goel ${ }^{3}$, Sanjeev Yadav ${ }^{4}$, Shreya Sharma ${ }^{5}$, Rohit K Sherawat ${ }^{6}$
}

\begin{abstract}
COVID pneumonia patient presents with fever, cough, and breathing difficulty. Many respiratory pathogens have such clinical presentations and pulmonary tuberculosis (PTB) is one of them, which is prevalent in the Indian subcontinent. Herein, we are presenting a case of dual infection with severe acute respiratory syndrome corona virus 2 (SARS-CoV-2) and drug-resistant PTB [likely multidrug resistance (MDR)] in a patient with chronic kidney disease (CKD) and type 2 diabetes mellitus, a clinical course further complicated by a prolonged viral clearance.

Keywords: Delayed viral clearance, Pulmonary tuberculosis, Severe acute respiratory syndrome corona virus 2.

Indian Journal of Critical Care Medicine (2020): 10.5005/jp-journals-10071-23662
\end{abstract}

\section{BACKGROUND}

COVID-19 is a global pandemic now that has taken away $>200,000$ lives globally. Early reports from China suggested that coinfection with another respiratory pathogen is rare. ${ }^{1}$ However, as the number of cases of COVID pneumonia is increasing day by day, more and more cases of dual infection with other respiratory pathogens are being reported. Pulmonary tuberculosis (PTB) and severe acute respiratory syndrome corona virus 2 (SARS-CoV-2) commonly involve the lungs and have similar clinical symptoms, such as, cough, fever, and breathing difficulty which may lead to a diagnostic dilemma.

\section{Case Description}

A 53-year-old woman came to the emergency department with complaints of breathing difficulty, fever, and cough for 6 days. She also had an episode of massive hemoptysis 4 days ago. Initially, she was treated at a hospital for 6 days, which later on referred her to a tertiary care hospital when her health deteriorated rapidly.

She was taking anti-tubercular therapy (ATT) for the last 1 month (HRZE Regimen) due to smear-positive pulmonary Koch. She is a known case of type 2 diabetes mellitus (DM) on oral hypoglycemic agents for the last 8 years. She was also diagnosed to have chronic kidney disease (CKD) stage 4.

The emergency department physician received her in a state of coma [glasgo coma scale (GCS): E1V1M1] with breathing difficulty. Her breathing pattern was deep and rapid ( $R R>25 /$ minute) with prominent use of accessory muscles of respiration. Oxygen saturation $\left(\mathrm{SpO}_{2}\right)$ could be maintained at $97 \%$ with $10 \mathrm{~L}$ oxygen/ min. Pupils were $3 \mathrm{~mm}$ in size and reacting to light.

She was intubated because of respiratory distress and poor GCS and shifted to COVID intensive care unit. Initial blood biochemistry revealed a normal total leukocyte count $\left(8,600 / \mathrm{mm}^{3}\right)$, neutrophil $86 \%$, lymphopenia (5\%), monocytosis (9\%), and hemoglobin 7.6 $\mathrm{g} / \mathrm{dL}$. Chest X-ray [anteroposterior (AP) view] showed bilateral lung consolidation which was followed up with high resolution computed tomography (HRCT) chest (Fig. 1A) that showed bilateral infiltrates. These infiltrates were much more predominant on the right side and consisted of an interstitial pneumonia pattern with mixed ground glass and consolidation giving the appearance of
1,4,5 Department of Critical Care and Pulmonology, Nayati Hospital, Mathura, Uttar Pradesh, India

${ }^{2}$ Department of Critical Care, Pulmonology and Sleep Medicine, Nayati Medicity, Noida, Uttar Pradesh, India

${ }^{3}$ Department of Internal Medicine, Nayati Medicity, Mathura, Uttar Pradesh, India

${ }^{6}$ Critical Care Department, Nayati Hospital, Mathura, Uttar Pradesh, India

Corresponding Author: Utpal Sarma, Department of Critical Care and Pulmonology, Nayati Hospital, Mathura, Uttar Pradesh, India, Phone: +918811079816, e-mail: drutpalrgci@gmail.com

How to cite this article: Sarma U, Mishra V, Goel J, Yadav S, Sharma S, Sherawat RK. COVID-19 Pneumonia with Delayed Viral Clearance in a Patient with Active Drug-resistant Pulmonary Tuberculosis. Indian J Crit Care Med 2020;24(11):1132-1134.

Source of support: Nil

Conflict of interest: None

a crazy pavement pattern on the right side. A round area of lung consolidation with air bronchograms, traction bronchiectasis with fibrosis are also seen in the right lung parenchyma. These two computed tomography (CT) findings are likely to be due to coexistent PTB.

She was started with Inj ampicillin, tab azithromycin, and vitamin C $500 \mathrm{mg}$ 8th hourly. Kidney function was grossly deranged with high serum creatinine and blood urea $(5.18$ and $190 \mathrm{mg} /$ $\mathrm{dL}$, respectively). Intravenous fluid resuscitation resulted in the production of urine and gradual reduction of serum creatinine. Total leukocyte count; kidney function test, and liver function test were regularly monitored. Serum lactate dehydrogenase (LDH), serum ferritin, and c reactive protein (CRP) were high ( $283 \mathrm{U} / \mathrm{L}, 795 \mathrm{ng} / \mathrm{mL}$, and $39.7 \mathrm{mg} / \mathrm{mL}$, respectively). It was monitored regularly, which followed a decreasing trend with the improvement of the patient's clinical condition. Tab hydroxychloroquine (HCQ) was started after the correction of serum electrolyte values.

Endotracheal (ET) secretion for Gram stain, aerobic culture, acid fast bacilli (AFB) satin, GeneXpert for TB, and AFB culture was undertaken. Respiratory tract polymerase chain reaction (RT-PCR) 

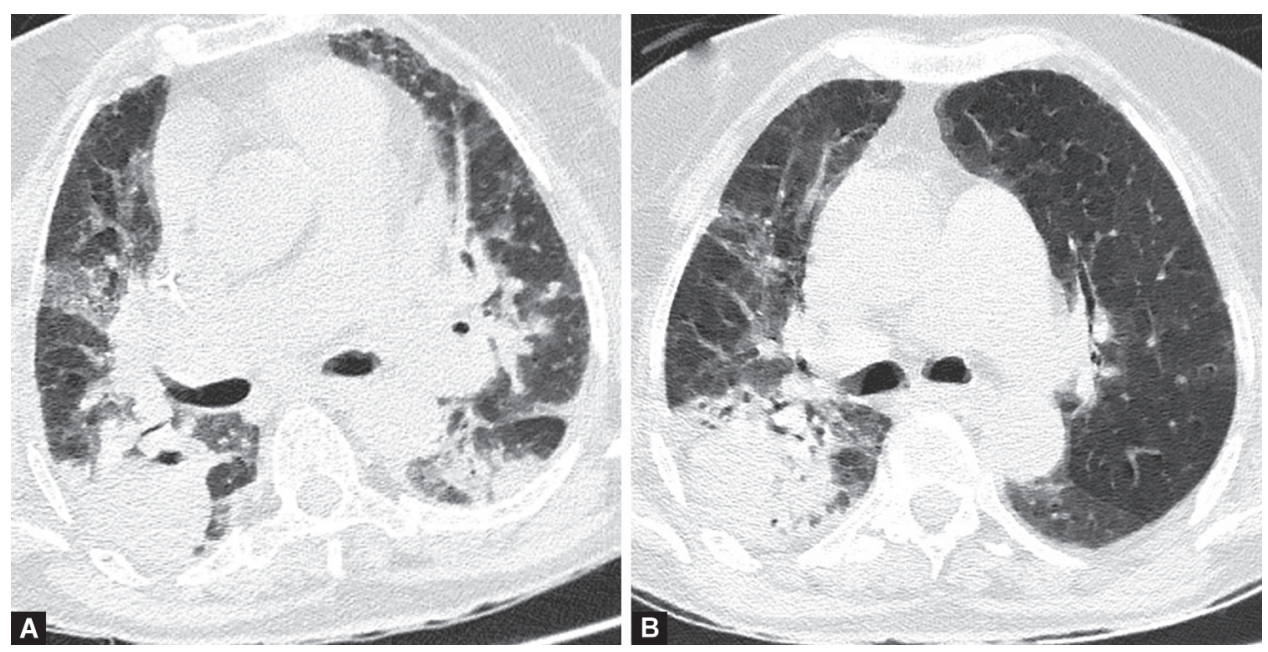

Figs $1 \mathrm{~A}$ and B: HRCT chest axial section (A) shows multilobar ground-glass opacity (GGO) on the day of admission and gradual resolution of GGO (B) after 4 weeks of treatment. Please note the round area of lung consolidation with few air bronchograms and traction bronchiectasis with fibrosis in the right lung parenchyma

for SARS-CoV-2 in ET secretion was positive. RT-PCR for H1N1 was negative. Tracheal secretion was positive for AFB stain. GeneXpert test showed rifampicin resistance. Multidrug resistance (MDR) regimen as per the $\mathrm{WHO}$ guidelines in a renal modification dose was started in view of coexistent CKD. AFB culture result is still awaited. Her blood sugar level was controlled by insulin infusion.

She was ventilated with volume control mode with a tidal volume of $8 \mathrm{~mL} / \mathrm{kg}$ of ideal body weight. Lung mechanics were within the normal range. Positive end expiratory pressure (PEEP) of $8 \mathrm{~cm}$ was applied. $\mathrm{FiO}_{2}$ was titrated with a target $\mathrm{SpO}_{2}$ of 94\%. Inj midazolam and inj fentanyl infusion were titrated as per the requirement. On day 5 , she was liberated from mechanical ventilation after a trial pressure support ventilation. Post-extubation she required oxygen support for 3 days.

A repeat HRCT chest (Fig. 1B) done on the 27th day of treatment showed a significant resolution of ground-glass opacity (GGO) in both lungs. However, the other image findings of fibrosis, bronchiectasis, and dense consolidation with air bronchograms remain. SARS-CoV-2 RT-PCR was repeated as per the local government health guidelines which continued to remain positive till the 44th day since the onset of symptoms. The patient was discharged after two consecutive negative results of RT-PCR for SARS-CoV-2.

\section{Discussion}

The COVID-19 pandemic continues to spread globally including the Indian subcontinent. The Indian subcontinent also has a huge burden of other infectious diseases like pulmonary Koch and tropical diseases.

Kim et al. ${ }^{2}$ in a recent study reported that the presence of a nonSARS-CoV-2 pathogen may not provide reassurance that a patient does not also have SARS-CoV-2. Our patient presented with cough, fever, and breathing difficulty which was initially presumed to be due to her existing illness (pulmonary Koch and CKD). Therefore, she was not investigated for SARS-CoV-2 in the facility where she was admitted for 6 days. Her clinical condition deteriorated rapidly and then transferred to our hospital which is a tertiary care facility in the state of Uttar Pradesh, India. We suspected COVID pneumonia based on her HRCT chest findings and ET secretions are sent for necessary tests to detect other respiratory pathogens. To date, there is no reported case of dual lung infection with drug-resistant TB and SARS-CoV-2.

Older age and certain comorbidities like diabetes mellitus, chronic obstructive pulmonary disease, and CKD increase the likelihood of severe COVID-19 and the necessity for intensive care and mechanical ventilation. ${ }^{3}$ These risk factors are also poor prognostic factors in PTB. Those patients who have lung damage from the past tuberculosis sequelae or chronic obstructive pulmonary disease may suffer from more severe illness if they are infected with COVID-19. There is thus a stronger case for concurrent testing for both conditions in these individuals even if the clinical picture is atypical.

HRCT chest has proven to be extremely useful in the diagnosis and assessment of the temporal progression of COVID-19 pneumonia. ${ }^{4,5}$ In our case, a prompt decision to do an HRCT chest on admission proved to be crucial in suspecting COVID pneumonia leading to its final diagnosis in RT-PCR. HRCT chest also helps to rule if there is any other lung pathology.

The coexistence of COVID pneumonia and MDR TB challenges the healthcare workers ( $\mathrm{HCW}$ ) and the infrastructure to provide for both droplet and airborne precautions. There is a moderate risk of cardiac arrhythmia as a result of the drug interaction of HCQ with levofloxacin which is used in the MDR ATT regimen.

COVID infection may lead to a thrombotic phenomenon that warrants antithrombotic medication. ${ }^{6}$ In our case, we did not start it right away as there was recent hemoptysis. Thromboprophylaxis was started after she was extubated, serum urea level started settling down, and there was no sign of hemoptysis.

Type 2 DM, male sex, invasive mechanical ventilation, and delayed hospital admission are the observed factors in which delayed viral clearance is reported. ${ }^{7}$ Association of rifampicin resistance TB as a risk factor of prolonged viral clearance is not available in the current literature. The index case took 44 days since the onset of the first symptom to convert to negative RT-PCR for SARS-CoV-2.

\section{Conclusion}

An early diagnosis of COVID-19 pneumonia is important in the care of people with PTB who are vulnerable to unfavorable outcomes, including death. With associated preexisting lung 
conditions HRCT chest is an extremely important diagnostic tool that can help to identify patterns of injury in coexisting lung pathology.

We intend to highlight our observation of exceptionally prolonged time taken for viral clearance in the patient with drugresistant PTB.

\section{Clinical Significance}

Pulmonary tuberculosis and COVID pneumonia may present similarly. It will not be surprising to have a dual infection of PTB and COVID pneumonia in a country where the burden of tuberculosis is high. HRCT chest has helped us to find out the presence of another infection. COVID pneumonia needs to be correctly diagnosed at admission especially in hospitals treating non-COVID patients as well to protect their patients and HCW.

\section{References}

1. Chen N, Zhou M, Dong X, Qu J, Gong F, Han Y, et al. Epidemiological and clinical characteristics of 99 cases of 2019 novel coronavirus pneumonia in Wuhan, China: a descriptive study. Lancet 2020;395(10223):507-513. DOI: 10.1016/S0140-6736(20)30211-7.

2. Kim D, Quinn J, Pinsky B, Shah NH, Brown I. Rates of co-infection between SARS-CoV-2 and other respiratory pathogens. JAMA 2020;323(20):2085-2086. DOI: 10.1001/jama.2020.6266.

3. Wang D, Hu B, Hu C, Zhu F, Liu X, Zhang J, et al. Clinical characteristics of 138 hospitalized patients with 2019 novel coronavirus-infected pneumonia in Wuhan, China. JAMA 2020;323(11):1061-1069. DOI: 10.1001/jama.2020.1585.

4. Ai T, Yang Z, Hou H, Zhan C, Chen C, Lv W, et al. Correlation of chest CT and RT-PCR testing in coronavirus disease 2019 (COVID-19) in China: a report of 1014 cases. Radiology 2020;296(2):E32-E40. DOI: 10.1148/ radiol.2020200642.

5. Caruso D, Zerunian M, Polici M, Pucciarelli F, Polidori T, Rucci C, et al. Chest CT features of COVID-19 in Rome, Italy. Radiology 2020;296(2):E79-E85. DOI: 10.1148/radiol.2020201237.

6. Klok FA, Kruip MJHA, van der Meer NJM, Arbous MS, Gommers DAMPJ, Kant KM, et al. Incidence of thrombotic complications in critically ill ICU patients with COVID-19. Thromb Res 2020;191:145-147. DOI: 10.1016/j.thromres.2020.04.013.

7. Xu K, Chen Y, Yuan J, Yi P, Ding C, Wu W, et al. Factors associated with prolonged viral RNA shedding in patients with COVID-19. Clin Infect Dis 2020;71(15):799-806. DOI: 10.1093/cid/ciaa351. 